\title{
CARNET DE LA RÉDACTION COMMENT FONCTIONNEMENT LES COMITÉS DE LECTURE
}

Depuis sa fondation même, La Revue canadienne du vieillissement doit sa haute tenue au travail des comités de lecture. Peut-être nos lecteurs aimeraient-ils connitre le fonctionnement de ces comités? Chaque revue a sa façon de procéder, et celle de La Revue canadienne du vieillissement est liee à la structure de son bureau. Précisions d'entrée de jeu que ses lecteurs s'efforcent toujours de se montrer justes, équitables et compréhensifs dans leurs critiques, et qu'ils tiennent également à faire oeuvre d'éducateurs. Si un certain nombre de manuscrits sont refusés, fort peu sont acceptés sans quelques révisions. Qu'il me soit donc permis de retracer ici les étapes par lesquelles passent la plupart des textes qui nous sont soumis.

Ceux-ci sont adressés au directeur en cinq exemplaires; ils doivent être conformes à l'un ou l'autre des deux protocoles publiés dans chaque livraison, au recto de la couverture à la fin de la revue. Si l'auteur n'a pas précisé la rubrique sous laquelle il souhaite faire paraître son article, le directeur choisit luimême un rédacteur adjoint qualifié pour apprécier le contenu de l'article. La revue s'est en effet donné un rédacteur adjoint pour chacun des domaines qui l'intéressent: Sciences de la santé, Sciences sociales, BienEtre social, Sciences biologiques, Psychologie.

En qualité de rédacteur en chef, je revois personnellement chaque manuscrit afin de m'assurer que celui-ci est conforme au protocole et répond aux exigences de haute tenue intellectuelle qui sont les nôtres. A cette étape, un certain nombre de manuscrits sont retournés à leur auteur pour cause d'insuffisance ou parce qu'ils ne sont pas conformes au protocole, mais le fait est quand même assez rare. En raison du temps et du travail qu'exige de la part des collaborateurs bénévoles la recension des manuscrits, il serait certes injuste d'exiger d'eux qu'ils examinent toutes les communications sans un tri préalable.
Supposons maintenant que le manuscrit a passé ce premier cap. Un exemplaire demeure au bureau du directeur et quatre sont acheminés au rédacteur adjoint, qui en fait une seconde lecture selon les mêmes critères que le directeur (dans chaque livraison, les noms des chefs de rubrique apparaissent à l'intérieur de la couverture au commencement de la revue). Si le rédacteur adjoint estime que le manuscrit ne mérite pas un examen critique plus poussé, il communique son opinion au directeur. En fait, presque tous les manuscrits font l'objet d'une troisième lecture. Ils sont donc transmis à deux membres du comité de lecture, mais seulement après avoir été "démarqués" : le nom de l'auteur et celui de l'institution à laquelle celui-ci appartient sont masqués ainsi que tout marque qui pourrait permettre d'en reconnaître la provenance.

L'idéal serait que les membres du comité de lecture ignorent totalement l'origine des manuscrits sousmis à leur appréciation, mais comme ces lectures sont choisis en raison de leur expertise dans le domaine concerné, il se peut qu'ils parviennent à la découvrir. Quoi qu'il en soit, les auteurs pour leur part ne sont jamais informés du nom des lecteurs, et l'anonymat permet à ces derniers d'exercer leurs fonctions de critiques en toute impartialité et objectivité.

Les lecteurs rédigent un rapport critique d'une page (plus souvent de quelques pages) et le font parvenir au rédacteur adjoint accompagné de l'une est mentions suivantes: 1) accepté, 2) accepté avec corrections mineures, 3) exige révision majeure, 4) refusé.

A la réception des deux rapports, le chef de rubrique compare ceux-ci afin d'en apprécier la concordance. Sill y découvre des divergences importantes, il fait appel à un troisième lecteur. Normalement, tous les manuscrits exigent quelques révisions. C'est le chef de rubrique qui en informe l'auteur, à qui il transmet les rapports critiques en y ajoutant ses propres 
commentaires et en soulignant les modifications qu'il juge les plus nécessaires.

Les manuscrits subissent ensuite en traitement quelque peu différent selon qu'ils ont reçu la cote 2 (corrections mineures) ou la cote 3 (révision majeure). Dans le premiers cas, les textes corrigés ne sont relus que par le rédacteur adjoint lors de leur seconde présentation. Dans le second cas-révision majeure -le texte révisé est renvoyé à l'un des membres du comité de lecture pour une seconde appréciation. La plupart des manuscrits revus et corrigés finissent par être acceptés, mais les délais sont certes moins longs lorsqu'il ne s'agit que de corrections mineures.

En fait, j'ai pu contaster que les retards attenants à l'appréciation des manuscrits sont le plus souvent dûs aux lenteurs des auteurs auxquels on demande une révision majeure. Pourtant, ceux-ci devraient comprendre que si le manuscrit est parvenu à ce stade de son examen, il sera très probablement accepté. Le même raisonnement vaut, à plus forte raison, pour les manuscrits qui ne nécessitent que des corrections mineures.

A la lumière des appréciations portées par les membres du comité de lecture, le rédacteur adjoint recommande au directeur d'accepter ou de refuser le manuscrit. Sa recommandation est toujours accompagnée des rapports rédigés par les membres du comité de lecture ainsi que de la correspondance à laquelle ont donné lieu les échanges avec l'auteur. Ces documents permettent en effet d'exercer un contrôle sur l'ensemble de l'opération. La décision finale revient au directeur de la revue.

La procédure semble sans doute fort complexe et quelque peu inquiétante. Elle ne vise pourtant qu'à garantir la qualité des articles qui paraissent dans La Revue canadienne $d u$ vieillissement. Or un article paru dans une revue dotée d'une comité de lecture a plus de mérite qu'un autre car il témoigne de l'estime des collègues pour son auteur, estime qui peut s'avérer déterminante au moment de demander une prime au mérite, une promotion, ou l'inamovibilité universitaire. Qu'un n'aille pas toutefois s'imaginer que nous nous accomplissons tout ce travail uniquement pour faciliter les promotions; notre intention première est d'assurer à la revue une collaboration de la plus haute qualité.

L'examen des manuscrits par les comités de lecture qualifés nous permet d'atteindre cet objectif non seulement en faisant obstacle aux communications médiocres mais aussi en contribuant à la formation des auteurs. Je suis en effet fort satisfait de la qualité des rapports critiques auxquels ont donné lieu les manuscrits soumis à La Revue canadienne $d u$ vieillissement et je suis persuadé qu'ils accomplissent une fonction éducative certaine. En effet, les membres de nos comités de lecture ainsi que nos rédacteurs adjoints donnent généreusement leur temps et leur énergie non seulement en vue de critiquer, mais aussi en vue d'instruire. Leur conduite est empreinte d'un altruisme semblable à celui du donneur de sang, qui sait que son don anonyme profitera à la collectivité. Et j'ajoute que les membres de nos comités de lecture ne reçoivent d'autre récompense que la satisfaction de voir paraittre leur nom dans la dernière livraison annuelle de la revue.

Le système des comités de lecture est sans doute encore bien imparfait, et je n'ignore pas que des articles ont été acceptés dans des revues de gérontologies fort respectées près avoir échoué à l'examen critique de certains de nos comités. Mais nous faisons de notre mieux et j'ose espérer que ce sont les critiques constructives formulées par les lecteurs anonymes et bénévoles de La Revue canadienne du vieillissement qui ont contribué à l'acceptation de ces manuscrits par d'autres revues.

Tous les articles qui paraissent dans $L a$ Revue canadienne du vieillissement sont soumis à un comité de lecture. Cette règle ne souffre AUCUNE EXCEPTION. Par contre, les Carnets de la rédaction ne sont pas revus par un comité de lecture, et les comptes rendus d'ouvrages ne sont relus que par le responsable des comptes rendus (qui se trouve actuellement être le directeur de la revue). De 
temps à autre, nous publierons des numéros spéciaux, comme le numéro sur les «Tendances en gérontologie et questions concernant le bien-être social» prévu pour le mois de mai 1987. Tous les manuscrits soumis en vue de ce numéro seront relus par des comités de lecture.

Le directeur, Victor W. Marshall 